\title{
The role of intestinal endotoxemia in a rat model of aluminum neurotoxicity
}

\author{
FENG WANG ${ }^{1}$, RUI-XIA GUO ${ }^{1}$, WEN-XING LI ${ }^{2}$, BAO-FENG YU ${ }^{3}$, BAI HAN ${ }^{4}$, LI-XIN LIU $^{5}$ and DE-WU HAN ${ }^{1}$ \\ ${ }^{1}$ Department of Pathophysiology, Key Laboratory of Cell Physiology of Ministry of Education, Shanxi Medical University, \\ Taiyuan, Shanxi 030001; ${ }^{2}$ Department of Surgery, The Second Hospital of Shanxi Medical University, \\ Taiyuan, Shanxi 030013; ${ }^{3}$ Department of Biochemistry and Molecular Biology, \\ Key Laboratory of Cell Physiology of Ministry of Education, Shanxi Medical University, \\ Taiyuan; ${ }^{4}$ Department of Psychiatry, The First Hospital of Shanxi Medical University; \\ ${ }^{5}$ The Experimental Center of Science and Research, The First Hospital, Institute of Hepatopathy, \\ Key Laboratory of Cell Physiology of Ministry of Education, Shanxi Medical University, Taiyuan, Shanxi 030001, P.R. China
}

Received March 14, 2016; Accepted March 28, 2017

DOI: $10.3892 / \mathrm{mmr} .2017 .6780$

\begin{abstract}
The present study aimed to investigate the effects of intestinal endotoxemia (IETM) in a rat model of aluminum neurotoxicity established by D-galactose and aluminum trichloride $\left(\mathrm{AlCl}_{3}\right)$. Adult Wistar rats were administered D-galactose and $\mathrm{AlCl}_{3}$ to create the aluminum neurotoxicity model. The learning and memory abilities of the rats were subsequently observed using a Morris water maze test and the serum levels of lipopolysaccharide (LPS), tumor necrosis factor (TNF)- $\alpha$, interleukin (IL)-1, diamine oxidase (DAO), glutamine (Gln) and glutaminase were measured. The expression of S-100 $\beta$ in the serum was detected using an enzyme-linked immunosorbent assay. The expression levels of the amyloid $\beta$-protein $(\mathrm{A} \beta)$ precursor (APP), presenilin 1 (PS1), $\beta$-site APP-cleaving enzyme (BACE), zona occludens protein (ZO)-1 and $\mathrm{A} \beta$ 1-40 in the brain of rats were detected via reverse-transcription polymerase chain reaction, western blotting and immunohistochemistry. The levels of LPS, TNF- $\alpha$, IL-1, DAO, Gln and $\mathrm{S}-100 \beta$ in serum and the mRNA and protein expression levels of APP, PS1, BACE and A $\beta 1-40$ in the brain were markedly increased in the model rats compared with controls. The level of glutaminase in the serum and the expression of ZO-1 in the brain were decreased in the model rats compared with controls. IETM was present in the rat model of aluminum neurotoxicity
\end{abstract}

Correspondence to: Dr De-Wu Han, Department of Pathophysiology, Key Laboratory of Cell Physiology of Ministry of Education, Shanxi Medical University, 56 Xinjian Southern Road, Taiyuan, Shanxi 030001, P.R. China

E-mail: 416671731@qq.com

Key words: intestinal endotoxemia, aluminum neurotoxicity, lipopolysaccharide, D-galactose, aluminum trichloride, amyloid $\beta$-protein established by $\mathrm{D}$-galactose and $\mathrm{AlCl}_{3}$ and may be important in the development of this neurotoxicity.

\section{Introduction}

The first reported case of aluminum toxicity in humans was from the early 1970s and following this, aluminum overexpression has been demonstrated to result in severe brain damage and neurodegeneration (1). Aluminum-induced neuronal injury is associated with the neuroinflammatory response $(2,3)$. Previous studies have indicated that the consumption of anti-inflammatory medication attenuates aluminum-induced functional neurotoxicity in rats (4).

Intestinal endotoxemia (IETM) is involved in liver failure, is associated with lipopolysaccharides (LPS) and has previously been demonstrated to exhibit a pathogenetic mechanism of initiation (5). Typically, mild cases of IETM often result in chronic hepatic failure (6). Inflammation induced by LPS under conditions of IETM is important in the development of numerous diseases $(7,8)$, however, if IETM-induced inflammation occurs during the development of aluminum neurotoxicity, remains to be elucidated.

The aim of the present study was to investigate the effect of inflammation induced by IETM in a rat model of aluminum neurotoxicity established using D-galactose and aluminum trichloride $\left(\mathrm{AlCl}_{3}\right)$. D-galactose was used due to its known neurotoxic effect (9). Following successful creation of the rat model of aluminum neurotoxicity, the learning and memory abilities of the rats were observed. The serum levels of LPS, tumor necrosis factor (TNF)- $\alpha$, interleukin (IL)-1, diamine oxidase (DAO), glutamine (Gln) and glutaminase were detected to evaluate the degree of IETM. The expression levels of amyloid $\beta$-protein (A $\beta$ ) precursor (APP), presenilin 1 (PS1), $\beta$-site APP-cleaving enzyme (BACE), zona occludens protein (ZO)-1 and $A \beta 1-40$ in the brain were observed, in addition to the total quantity of lysozyme (LYZ) in the liver and S-100 $\beta$ in the serum of the rats. The results of the present study reveal the underlying role of LPS in the development of aluminum 
toxicity and thus, may provide novel strategies for the prevention and treatment of associated clinical diseases such as Alzheimer's disease and amyotrophic lateral sclerosis.

\section{Materials and methods}

Experimental protocol and ethics statement. Rats were provided by Vital River Experimental Technology Company Ltd. (Beijing, China). A total of 48 adult (age, 9-11 weeks) male Wistar rats, weighing 200-250 g, were housed 6 per cage at $20-25^{\circ} \mathrm{C}$ with a relative humidity of $50-60 \%$, a $12 \mathrm{~h}$ light-dark cycle and free access to food and water. Rats were randomized to either receive vehicle $\left(0.9 \% \mathrm{NaCl} ; 85 \mathrm{mg} \cdot \mathrm{kg}^{-1} \cdot \mathrm{day}^{-1}\right)$ or D-galactose intraperitoneal injection $\left(60 \mathrm{mg} \cdot \mathrm{kg}^{-1} \cdot \mathrm{day}^{-1}\right)$ and $\mathrm{AlCl}_{3}$ (25 mg.kg- $\left.\mathrm{kay}^{-1}\right)$ for 90 days, to establish the aluminum neurotoxicity model. Following this, the learning and memory abilities of the rats were observed using a Morris water maze. The serum levels of LPS, TNF- $\alpha$, and IL-1 were determined by the chromogenic Limulus amoebocyte lysate (CLAL) and radioimmunity assays. The level of DAO was measured using a Diamine oxidase colorimetric assay kit (Shanghai Bairui Biotech Co., Ltd., Shanghai, China) according to the manufacturer's instructions. The levels of Gln were measured using a Glutamine Colorimetric Assay kit (Biovision, Inc., Milpitas, CA, USA), according to the manufacturer's instructions, and glutaminase was measured using the Glutaminase ELISA assay kit (cat. no. 201233721; Cusabio Biotech Co., Ltd., Wuhan, Hubei, China) according to the manufacturer's instructions. All experimental animal procedures were approved by the Laboratory Animal Use and Care Committee of Shanxi Medical University (Taiyuan, China; permit no. SXMU-2012-18; ) and the Ethics Committee of Animal Experiments of Shanxi Medical University (permit no. 20120623-1). All surgery was performed under sodium pentobarbital anesthesia and all possible efforts were made to minimize the suffering of the experimental rats. The rats were sacrificed via anesthetic overdose.

Morris water maze. The Morris water maze was used to test the learning and memory of the rats for 5 days ( $n=10 /$ group) and was performed 8 weeks following administration (10). For this experiment, a circular stainless steel tank $(155 \mathrm{~cm}$ in diameter and $60 \mathrm{~cm}$ deep) containing water $\left(24-26^{\circ} \mathrm{C}\right)$ to a depth of $40 \mathrm{~cm}$ was used; the extramaze cues, which consisted of four uniquely colored and textured inserts (coarse plastic mesh, metal screen, ridged plastic and sandpaper) placed on the arms of the plus-maze, were kept constant. The rats learned to escape from the water by searching for a submerged, and therefore hidden, Plexiglas platform $(10 \mathrm{~cm}$ in diameter and $38.5 \mathrm{~cm}$ high), which was kept in a fixed position. To familiarize them with the experimental situation, prior to the experiment, the rats swam for $90 \mathrm{sec}$ with the platform removed. During each trial, the animal was placed in the water facing the wall at one of five designated starting points. Animals that failed to find the platform within $60 \mathrm{sec}$ were placed onto the platform and kept there for $30 \mathrm{sec}$, then the rats were removed from the maze until the next repeat of the experiment. The time taken to find the platform, termed the escape latency, was measured. This test was performed once for each of the 5 starting points per day for 5 days.
Clal. The CLAL test kit was obtained from Chromogenix (cat. no. 20120056; Molndal, Sweden). The analysis was conducted according to the manufacturer's protocol. The coefficient of variation was $8 \%$ ( $n=10$ /group).

Immunohistochemistry. The expression of LYZ in the liver and $A \beta 1-40$ in the brain (hippocampus) were detected via immunohistochemistry (11-13). Rabbit anti-rat/-rabbit LYZ and A $\beta 1-40 \mathrm{IgG}$ antibodies (Abcam, Shanghai, China) were used. Following the completion of the Morris water maze experiments, 12 rats ( 6 rats/group) were sacrificed and the whole brain and liver were removed and dissected. Prior to sectioning, liver tissue and hippocampus samples were fixed in $10 \%$ formalin for $24 \mathrm{~h}$ at room temperature, then embedded in paraffin. The paraffin blocks were cooled to $-10^{\circ} \mathrm{C}$ to ease sectioning. Using the Microm HM340 E microtome (Thermo Fisher Scientific Inc., Waltham, MA, USA), 4- $\mu \mathrm{m}$ sections were generated. The EnVision ${ }^{\text {TM }}$ staining kit was used according to the manufacturer's instructions, for immunohistochemical analyses [GI2 Doublestain System, Rabbit/Mouse (DAB+/Permanent RED); cat. no. K5361; Dako DK; Agilent Technologies, Inc., Santa Clara, CA, USA]. The optimal antigen retrieval method and concentration to use were determined per antibody. Following deparaffinization in dimethylbenzene twice at room temperature for 5 min each time, the slides were washed in PBS containing $0.05 \%$ Tween-20 prior to antigen retrieval in the microwave (for liver tissues; $2 \mathrm{~min} 780 \mathrm{~W}, 10 \mathrm{~min} 150 \mathrm{~W}$ and $10 \mathrm{~min}$ $80 \mathrm{~W}$ ) or in $70 \%$ formic acid (for hippocampus samples). The slides were placed in cuvettes, as a part of the capillary gap staining method (Thermo Fisher Scientific, Inc.), which was performed as described previously (14). Endogenous peroxidase activity was blocked by the EnVision ${ }^{\mathrm{TM}}$ dual endogenous enzyme block (Agilent Technologies, Inc.). Following washing with PBS containing $0.05 \%$ Tween-20, a second blocking step was applied using PBS, $0.1 \%$ bovine serum albumin (BSA; Sigma Aldrich; Merck KGaA, Darmstadt, Germany), $1 \%$ NHS and $0.2 \%$ Triton X-100. Then, the primary antibodies [rabbit anti-rat/-rabbit LYZ (cat. no. 20120789) and A $\beta 1-40$ (cat. no. 20123349) IgG antibodies; Abcam, Shanghai, China; with a $1: 3,000$ dilution in $0.1 \% \mathrm{BSA} / \mathrm{PBS}$ ] were added for $10 \mathrm{~min}$ at room temperature, followed by washing with PBS containing $0.05 \%$ Tween-20 and the addition of the EnVision ${ }^{\mathrm{TM}}$ polymer/horseradish peroxidase (HRP) goat anti-rabbit secondary antibody (cat. no. 201213219; 1:500 dilution; Dako Cytomation; Agilent Technologies, Inc.) for $10 \mathrm{~min}$ at room temperature. Following washing with $\mathrm{PBS}, \mathrm{DAB}+$ (from EnVision $^{\mathrm{TM}}$ staining kit; Dako DK; Agilent Technologies, Inc.) was added to initiate the enzymatic reaction and allow for visualization of the elements. A total of 10 high magnification fields were randomly selected from each slice and the number of positive cells per high magnification field (upright light microscope; CX31-LV320; Olympus Corporation, Tokyo, Japan) were counted. Following this, the average number of positive cells per slice was calculated.

Enzyme-linked immunosorbent assay (ELISA). The S-100 $\beta$ level was measured using an S-100 $\beta$ ELISA kit (cat. no. 20120166; Neogen Europe, Ltd., Lansing, MI, USA) according to the manufacturer's protocol ( $n=10 /$ group). 

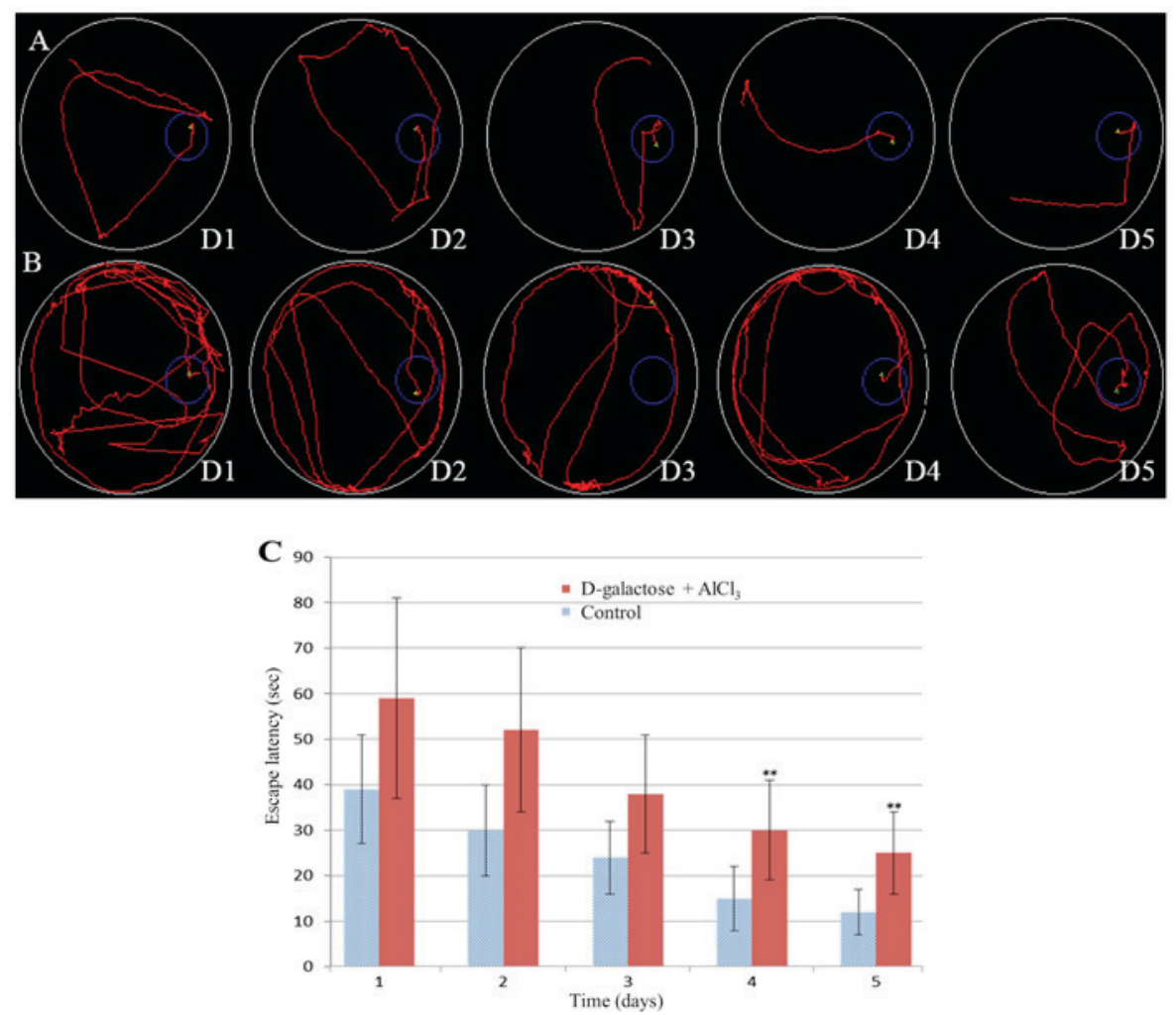

Figure 1. Effect of D-galactose and $\mathrm{AlCl}_{3}$ on cognitive impairment in rats. Swim path of (A) control group and (B) aluminum neurotoxicity model group. (C) Rat escape latency observed in the Morris water maze, from days 1-5. Sec, sec; D, day; $\mathrm{AlCl}_{3}$, aluminum trichloride. ${ }^{* *} \mathrm{P}<0.01$ vs. control group.

Western blotting. Protein was extracted from fresh brain tissue using a ProteoExtract Subcellular Proteome Extraction kit (Sigma Aldrich; Merck KGaA). Protein concentration in the lysates was evaluated using a bicinchoninic acid protein assay kit according to the manufacturer's instructions (Thermo Fisher Scientific, Inc.). Following 10\% sodium dodecyl sulfate polyacrylamide gel electrophoresis ( $5 \mu \mathrm{g}$ protein applied) and transfer onto a nitrocellulose membrane, the membranes were blocked with 5\% BSA (Sigma Aldrich; Merck KGaA) and $0.05 \%$ Tween-20 in Tris-buffered saline (TBST) for $1 \mathrm{~h}$ at room temperature. This was followed by overnight incubation at room temperature with the following primary antibodies: Monoclonal rabbit anti-rat ZO-1 antibody (1:200; cat. no. 201212315; Abcam), monoclonal rabbit anti-rat APP antibody (1:500; cat. no. 20121232; Abcam), monoclonal rabbit anti-rat PS-1 antibody (1:1,000; cat. no. 20123343; Abcam), monoclonal rabbit anti-rat BACE1 antibody $(1: 500$; cat. no. 201233561; Abcam) and monoclonal rabbit anti-rat $\beta$-actin antibody (1:1,000, cat. no. 201214231; Abcam). Following three washes with TBST, the membranes were incubated with a HRP-conjugated anti-rabbit antibody (1:5,000; Santa Cruz Biotechnology, Inc., Dallas, TX, USA) for $2 \mathrm{~h}$ at room temperature. The membranes were exposed to film (Kodak, Rochester, NY, USA) and developed. The films were scanned and analyzed using the Kodak IS440 Automated Digitizing System and image analytical software (ImageQuant TL v2005; GE Healthcare Life Sciences, Uppsala, Sweden) for densitometric analysis. Experiments were repeated 3 times.

Reverse-transcription polymerase chain reaction (RT-PCR). Primers: APP (295 bp), forward 5'-GGATGCGGAGTTCGG
ACATG-3' and reverse 5'-GTTCTGACTCTGCTCAAAG-3'; PS-1 (186 bp), forward 5'-AGATGCCTCCTCTGTCCTCA-3' and reverse 5'-CCGTCTTTGGGCATACATCT-3'; BACE1 (309 bp), forward 5'-CACCGAGACCGACGAAGAGCC-3' and reverse 3'-CTAATAGGCTATGGTCATGAGGGT-3'; GAPDH (307 bp), forward 5'-TTGCCAGTTGCTTTAGTG ATA-3' and reverse 5'-CTTTTTCCCCCATTTCATTTC-3'.

Total RNA from fresh brain tissue samples was extracted using the TRIzol ${ }^{\circledR}$ reagent (Takara Biotechnology Co., Ltd., Dalian, China) according to the manufacturer's instructions. RNA was reverse transcribed to cDNA using the Takara PrimeScript 1st Strand cDNA Synthesis kit, according to the manufacturer's instructions, and the obtained cDNA was used as a template to perform PCR amplification. Semi-quantitative RT-PCR was performed with a mixture of the cDNA product, primers, dNTP and Taq DNA polymerase (Invitrogen; Thermo Fisher Scientific, Inc.). The thermocycling conditions used were as follows: $95^{\circ} \mathrm{C}$ for $30 \mathrm{sec}, 55^{\circ} \mathrm{C}$ for $30 \mathrm{sec}$ and $72^{\circ} \mathrm{C}$ for $30 \mathrm{sec}$ for 25 cycles, followed by a final extension at $72^{\circ} \mathrm{C}$ for $5 \mathrm{~min}$. Results were normalized to the APP reference gene. Electrophoresis was performed on $1.5 \%$ agarose gel $(10 \mathrm{~mA}$; $100 \mathrm{~V} ; 30 \mathrm{~min})$. The images were scanned and then analyzed with Multi-Analyst software (Bio-Rad Laboratories, Inc., Hercules, CA, USA).

Statistical analysis. Data were analyzed using the SPSS software version 22.0 (IBM Corp., Armonk, NY, USA). All values are expressed as the mean \pm standard deviation. Statistical analysis was performed using an unpaired Student's t-test, multiple-factor repetitive measurement and one-way repeated measures analysis of variance (for learning and memory 
A

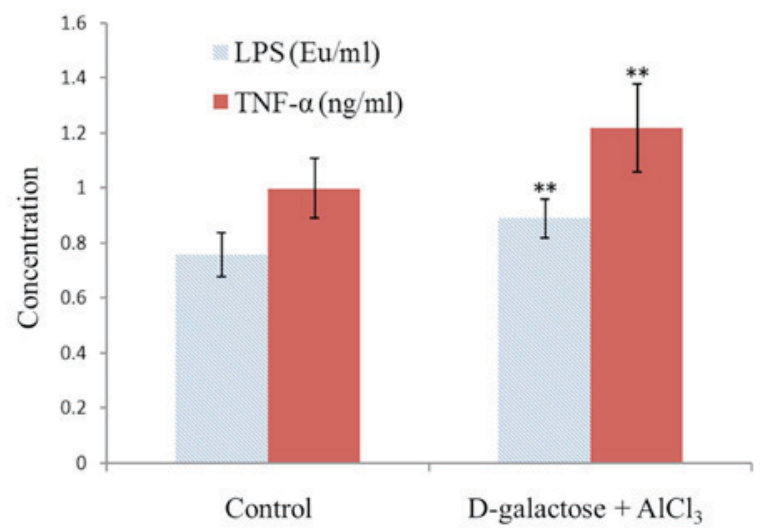

B

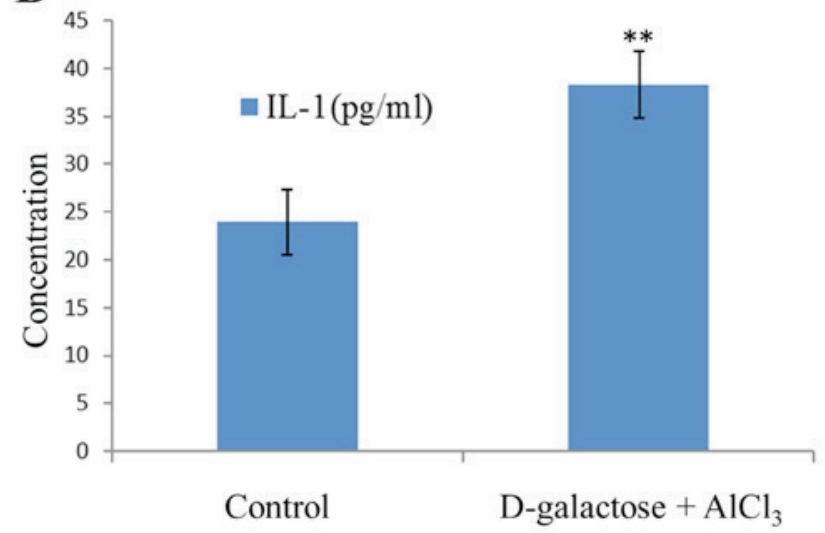

Figure 2. Concentrations of the LPS, TNF- $\alpha$ and IL-1 in rat plasma. (A) LPS and TNF- $\alpha$ levels in the control and model groups. (B) Level of IL-1 in the control and model groups. LPS, lipopolysaccharide; TNF- $\alpha$, tumor necrosis factor- $\alpha$; IL, interleukin. ${ }^{* *} \mathrm{P}<0.01$ vs. control group.

abilities). $\mathrm{P}<0.05$ was considered to indicate a statistically significant difference.

\section{Results}

Learning and memory abilities. The rat escape latency in the Morris water maze from days $1-5$ was $59 \pm 22$, $52 \pm 18,38 \pm 13$, $30 \pm 11$ and $25 \pm 9 \mathrm{sec}$, respectively, in the D-galactose $+\mathrm{AlCl}_{3}$ group and $39 \pm 12,30 \pm 10,24 \pm 8,15 \pm 7$ and $12 \pm 5 \mathrm{sec}$, respectively, in the control group (Fig. 1A and B). These data are additionally presented in Fig. 1C. There were no differences in the two groups from days 1-3 ( $>>0.01)$. However, from days 4-5, the escape latency in the D-galactose $+\mathrm{AlCl}_{3}$ group was increased compared with the control group $(30 \pm 11$ vs. $15 \pm 7 \mathrm{sec}$ and $25 \pm 9$ vs. $12 \pm 5 \mathrm{sec}$, respectively; $\mathrm{P}<0.01$ ). From days $4-5$, the swim path of the control group was altered from a random line to a straight line. However, the swim path of the test group remained random (Fig. 1B). The memory ability was decreased in the rats treated with $\mathrm{D}$-galactose and $\mathrm{AlCl}_{3}$.

Levels of TNF- $\alpha, I L-1$, and LPS. The geometric means of the LPS, TNF- $\alpha$ and IL-1 levels in the normal rats were $0.76 \pm 0.08 \mathrm{EU} / \mathrm{ml}, 1.0 \pm 0.11 \mathrm{ng} / \mathrm{ml}$ and $23.96 \pm 3.39 \mathrm{pg} / \mathrm{ml}$, respectively. However, those in the test group were $0.89 \pm 0.07 \mathrm{EU} / \mathrm{ml}$, $1.22 \pm 0.16 \mathrm{ng} / \mathrm{ml}$ and $38.38 \pm 3.48 \mathrm{pg} / \mathrm{ml}$, respectively, with a significant difference observed $(\mathrm{P}<0.01$; Fig. 2$)$.

Expression of LYZ in liver. There were numerous brown LYZ cells in the control rat livers (30.6 $\pm 8.2 ; n=10)$, however fewer LYZ cells were present in the livers of the aluminum neurotoxicity model rats $(18.1 \pm 5.1 ; \mathrm{n}=10 ; \mathrm{P}<0.01$ vs. control group; Fig. 3$)$. This observation indicated decreased Kupffer cell function.

Intestinal mucosal barrier function. The levels of DAO and Gln in the serum and intestinal mucosa of the model rats were significantly greater compared with control rats $(\mathrm{P}<0.01)$, whereas the level of glutaminase was decreased in the model rats compared with control rats $(\mathrm{P}<0.01$; Table I).

Blood-brain barrier (BBB) function. The expression of $\mathrm{S}-100 \beta$ in the serum $(0.33 \pm 0.18)$ was increased in the model rats compared with control rats $(0.11 \pm 0.03 ; \mathrm{n}=10 ; \mathrm{P}<0.01)$. The expression of ZO-1 in the brain was increased in the control rats $(53.3 \pm 8.4$; ratio of $Z O-1 / \beta$-Actin) compared with the model rats $(26.9 \pm 5.6 ; \mathrm{P}<0.01$; Fig. 4). These data demonstrated that the BBB function was decreased and permeability was increased in the model group.

mRNA and protein expression levels of APP, PS and BACE1. As presented in Fig. 5, the addition of D-galactose and $\mathrm{AlCl}_{3}$ resulted in a marked increase in the expression of APP, PS1 and BACE1 mRNA $(72 \pm 12,52 \pm 10$ and $72 \pm 15 \%$, respectively) that were $\sim$ two-fold increases compared with control rats $(30 \pm 6$, $32 \pm 7$ and $36 \pm 9 \%$, respectively). The protein expression levels of APP, PS1 and BACE1 in the model and control groups demonstrated a similar trend to the aforementioned mRNA results.

Expression of A $\beta 1-40$. As presented in Fig. 6, the expression of $A \beta 1-40$ in the hippocampal brain of the aluminum neurotoxicity model rats $(24.1 \pm 5.1)$ was increased compared with control rats $(8.6 \pm 2.2 ; \mathrm{n}=10 ; \mathrm{P}<0.05$; Fig. $6 \mathrm{C})$.

\section{Discussion}

The pathological presentation of aluminum neurotoxicity involves regionalized neuronal dysfunction/apoptosis, lesions termed neurofibrillary tangles and deposition of $\mathrm{A} \beta$ protein $(15,16)$. In previous studies, hypotheses have been proposed to link the lesions and cytopathology of aluminum neurotoxicity with the development of inflammation (2). Inflammation has previously been suggested to act as a marker and potential inducer of aluminum neurotoxicity (17).

Han (5), Han (6) and Zhao and Han (7) first suggested that liver injury may result from increased levels of LPS. Advanced cases of IETM typically result in excessive inflammation with severe hepatic necrosis, hepatitis and acute liver failure. The human endotoxin model, an in vivo model of systemic inflammation in which LPS is injected or infused intravenously into healthy volunteers, may potentially be of use in elucidating the underlying mechanisms involved (18).

Cerebral deposition of $A \beta$ is a feature of aluminum neurotoxicity. Induction of $\mathrm{A} \beta$ overexpression via $\mathrm{AlCl}_{3}$ 
Table I. Levels of DAO, glutaminase and Gln in sera and intestinal mucosa.

\begin{tabular}{lcccc}
\hline & & \multicolumn{2}{c}{ Gln } \\
\cline { 4 - 5 } Group & DAO $(\mathrm{U} / \mathrm{ml})$ & Glutaminase $(\mathrm{mmol} / \mathrm{h} . \mathrm{g})$ & Sera $(\mathrm{mg} / \mathrm{dl})$ & Intestinal mucosa $(\mathrm{mg} / \mathrm{g})$ \\
\hline Control & $0.52 \pm 0.13$ & $2.37 \pm 0.34$ & $50.37 \pm 16.85$ & $3.28 \pm 1.24$ \\
D-galactose $+\mathrm{AlCl}_{3}$ & $1.18 \pm 0.36^{\mathrm{a}}$ & $1.24 \pm 0.31^{\mathrm{a}}$ & $196.54 \pm 4357^{\mathrm{a}}$ & $16.54 \pm 6.27^{\mathrm{a}}$ \\
\hline
\end{tabular}

Data are presented as the mean \pm standard deviation $(\mathrm{n}=10) .{ }^{\mathrm{a}} \mathrm{P}<0.01$ vs. control. DAO, diamine oxidase; Gln, glutamine; AlCl3, aluminum trichloride.

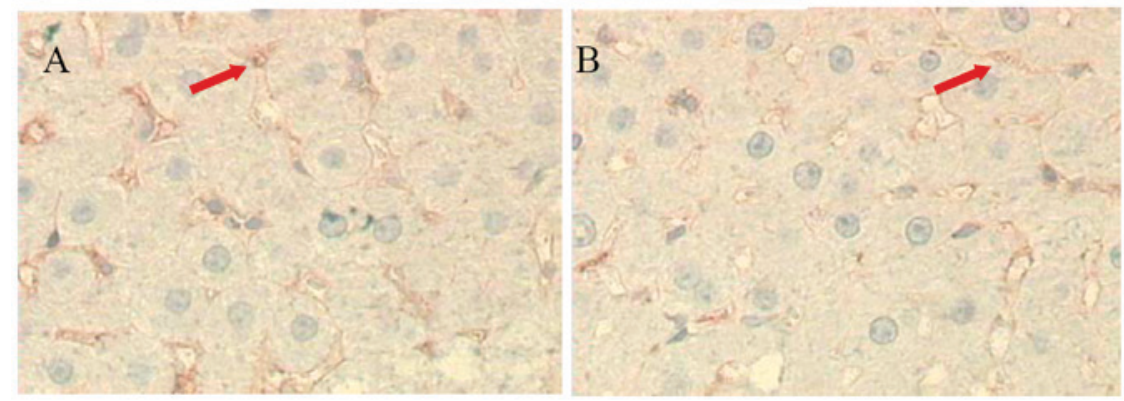

Figure 3. Expression of lysozyme in the liver (indicated by red arrows). Tissue sections obtained from (A) control and (B) aluminum neurotoxicity model rats, detected via immunohistochemistry. Magnification, x400.

$\mathbf{A}$

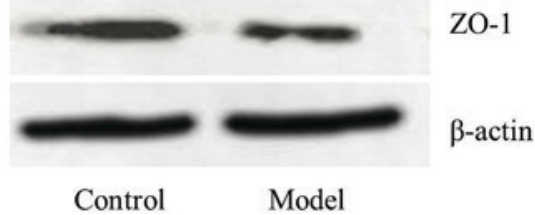

B

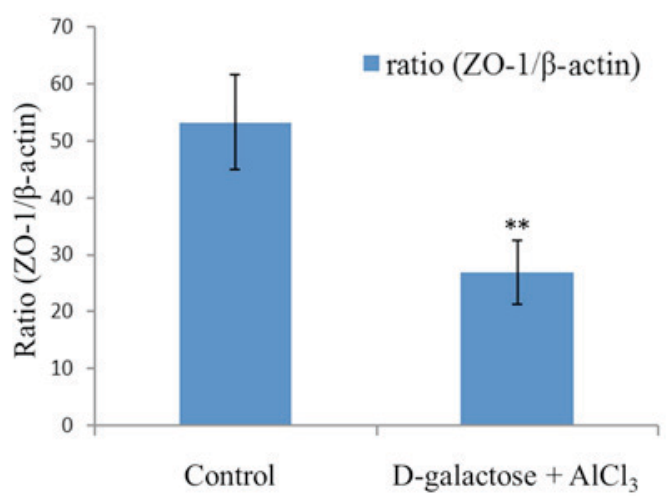

Figure 4. Expression of ZO-1 in the brain, detected via western blotting. (A) Western blotting was performed to evaluate the expression of ZO-1 in the brain. (B) The ratio of ZO-1/ $\beta$-actin. ZO-1, zona occludens protein; Model, aluminum neurotoxicity model. ${ }^{* *} \mathrm{P}<0.01$ vs. control group.

or D-galactose in the rat or mouse brain results in animal models of aluminum neurotoxicity and aging. Aluminum and $D$-galactose affect the expression of $A \beta$ metabolism-associated molecules, suggesting that this mouse model may be useful for studying the mechanisms and biomarkers of aluminum neurotoxicity (9).
In the present study, the escape latency of rats treated with D-galactose and $\mathrm{AlCl}_{3}$ was increased compared with the control group. The model rats exhibited a longer escape latency to find the platform when compared with the control rats. D-Galactose and $\mathrm{AlCl}_{3}$ resulted in increased mRNA and protein expression levels of APP, PS1 and BACE. The expression of A $\beta$ was additionally increased. These results verify those from previous reports that suggest high doses of D-galactose and $\mathrm{AlCl}_{3}$ are associated with neurotoxicity (9). To the best of our knowledge, the present study demonstrated for the first time that the levels of LPS, TNF- $\alpha$ and IL-1 in the blood of rats treated with D-galactose and $\mathrm{AlCl}_{3}$ were significantly increased compared with the control group.

LPS is a potent inducer of inflammatory cytokines including TNF- $\alpha$, IL- 6 and IL-1 $\beta$ in macrophages and microglia (19-21). Bacterial LPS induces numerous host responses that are beneficial and detrimental (22). Liu et al (23) demonstrated that cluster of differentiation 14 interacts with $\mathrm{A} \beta$ fibrils and therefore contributes to microglial phagocytosis of $A \beta 42$ fibrils. Inflammation potentially increases the level of $A \beta$ present in the brain via three potential mechanisms: Increased influx, decreased efflux and increased neuronal production (24).

TNF- $\alpha$ is a primary pro-inflammatory cytokine and its role in the pathogenesis of aluminum neurotoxicity has previously been investigated (25). TNF- $\alpha$ influences the expression or metabolism of various molecules that are involved in the development of aluminum neurotoxicity, including $A \beta(26,27)$. $\mathrm{TNF}$ is upregulated in the mouse brain following exposure to aluminum (28).

IL-1, which is an immune regulatory cytokine, may promote the merging and secretion of APP (29) and A $\beta$ (30). Anti-inflammatory drugs have previously been suggested as 

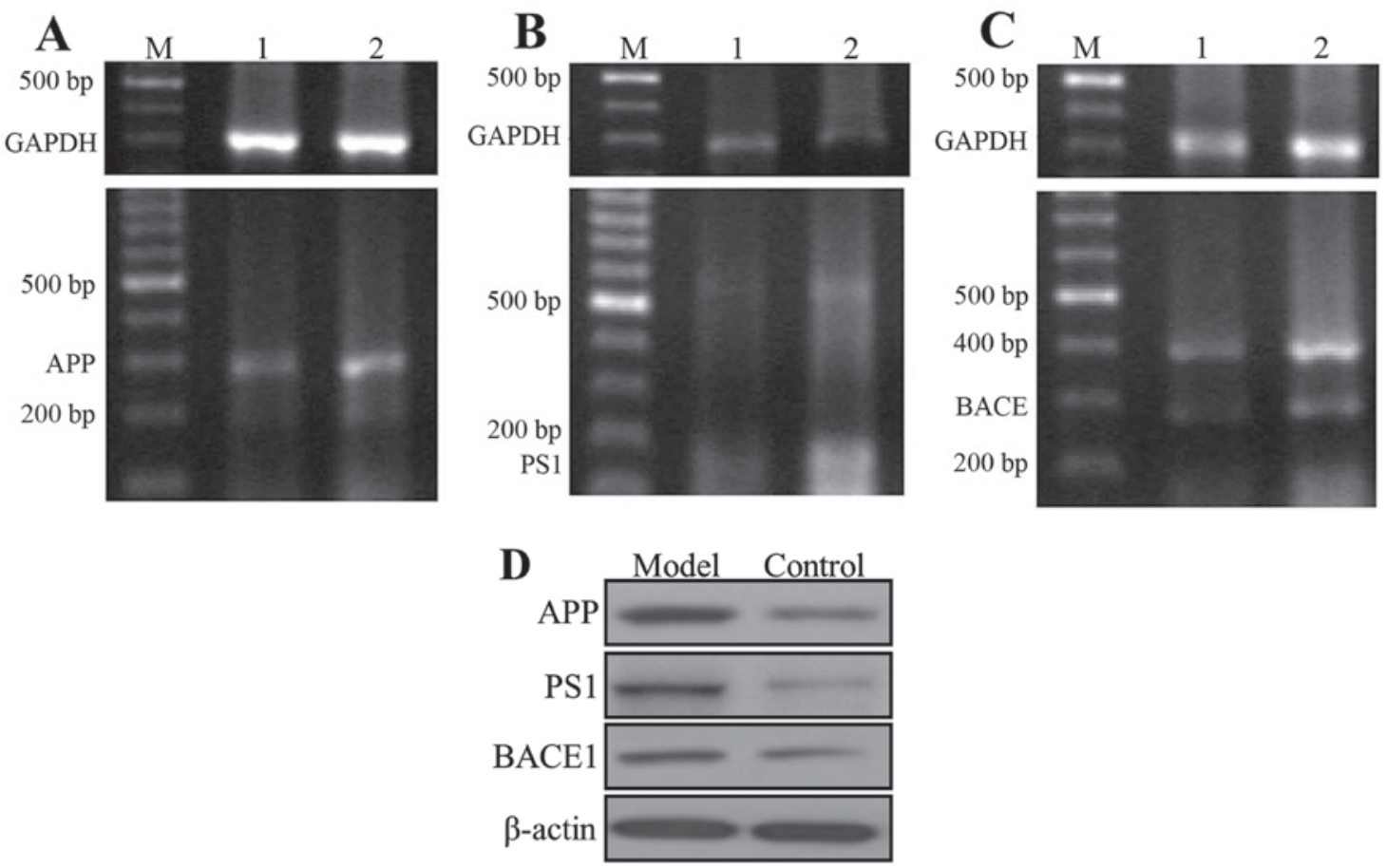

Figure 5. mRNA and protein expression levels of APP, PS1 and BACE1 in the rat brain. Expression of (A) APP, (B) PS1 and (C) BACE1 mRNA in the brain detected via reverse transcription-polymerase chain reaction. (D) Expression of APP, PS1 and BACE1 protein in the brain detected via western blotting. M, DNA molecule marker; 1 , control group; 2, aluminum neurotoxicity model group; bp, base pairs. APP, amyloid $\beta$-protein precursor; PS1, presenilin 1; BACE, $\beta$-site APP-cleaving enzyme.
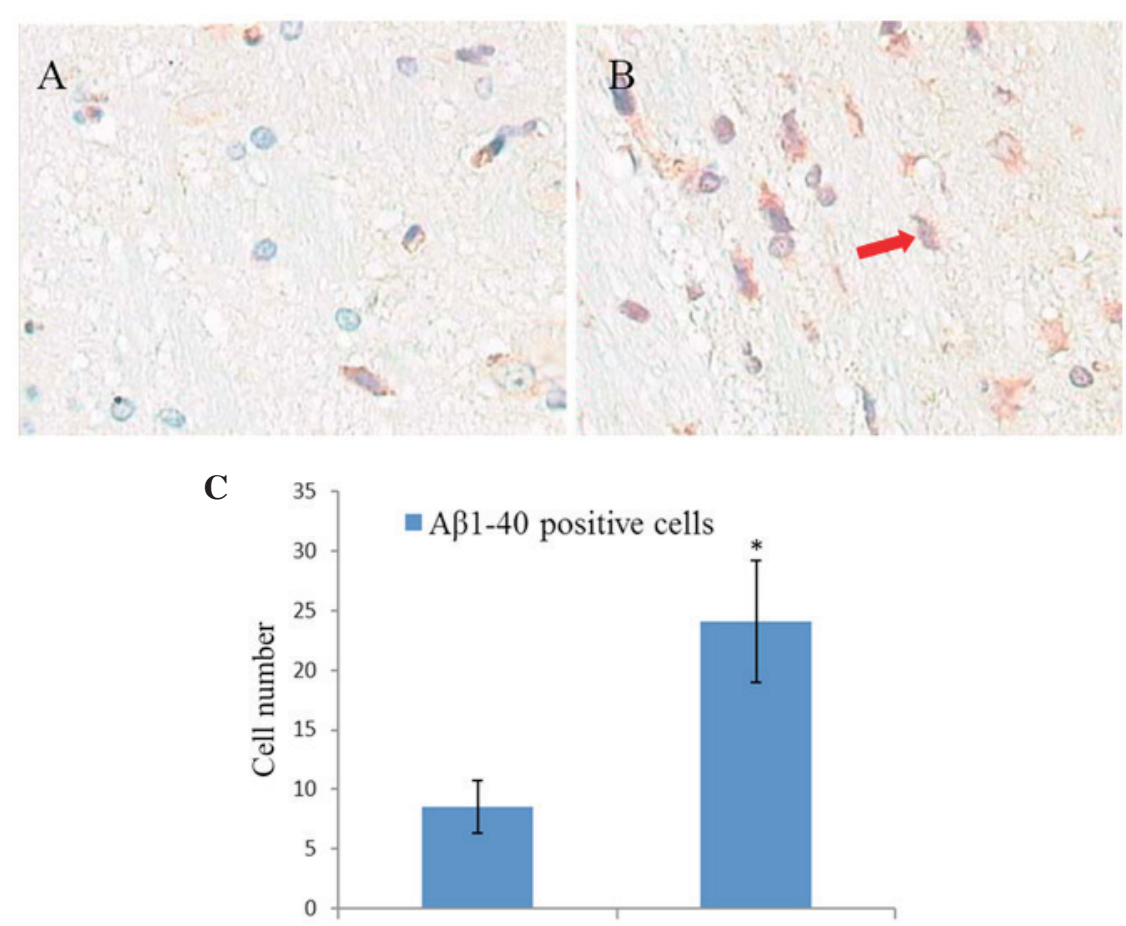

Figure 6. Expression of A $\beta 1-40$ in the rat brain. (A) Control and (B) aluminum neurotoxicity model A $\beta 1-40$ expression, detected via immunohistochemistry. The brown particles indicated by the red arrow are A $\beta 1-40$ positive cells (magnification, $\mathrm{x} 200$ ). (C) Expression of A $\beta 1-40$ in the rat brain. ${ }^{*} \mathrm{P}<0.05$ vs. control group. $A \beta$, amyloid $\beta$-protein.

a treatment for aluminum neurotoxicity. Tsunoda et al (28) suggests that IL-1 $\beta$ may be involved in one of the key disease mechanisms for aluminum neurotoxicity. Of all the numerous inflammatory cytokines associated with aluminum neurotoxicity, IL-1 $\beta$ in particular, has been identified to exhibit an important pathogenic role (27).

Furthermore, the Kupffer cell function in the model rat liver appeared to be decreased and the barrier function of 
the intestinal mucosa and BBB exhibited damage. A previous study suggested that aluminum may stimulate $\mathrm{Fe}^{2+}$-supported lipid peroxidation by binding to the membrane, thus affecting the BBB permeability (31). During BBB maturation and alteration, the brain endothelia develop a functional polarity and the membrane glycoprotein containing $\alpha$-D-galactose is downregulated (32). Therefore, the LPS from the intestinal mucosa are able to penetrate the intestinal mucosal barrier and enter the serum. In addition, the impaired function of Kupffer cells leads to decreased removal of plasma LPS. The high level of LPS subsequently activates the Kupffer cells, releasing TNF- $\alpha$ and IL- 1 and finally leads to IETM. The BBB damage allows LPS to readily enter the brain and induce inflammation.

In conclusion, the present study demonstrated that IETM was present in the rat model of aluminum neurotoxicity established by $\mathrm{D}$-galactose and $\mathrm{AlCl}_{3}$ and may be important in the development of neurotoxicity.

\section{Acknowledgements}

The authors would like to thank Mr. Yuanchang Zhao and Mrs. Jiahui Zhao (Department of Pathophysiology, Shanxi Medical University, Taiyuan, Shanxi, China) for their technical assistance.

\section{References}

1. Zhang Q, Li N, Jiao X, Qin X, Kaur R, Lu X, Song J, Wang L, Wang J and Niu Q: Caspase-3 short hairpin RNAs: A potential therapeutic agent in neurodegeneration of aluminum-exposed animal model. Curr Alzheimer Res 11: 961-970, 2014.

2. Campbell A: The role of aluminum and copper on neuroinflammation and Alzheimer's disease. J Alzheimers Dis 10: 165-172, 2006.

3. Castorina A, Tiralongo A, Giunta S, Carnazza ML, Scapagnini G and D'Agata V: Early effects of aluminum chloride on beta-secretase mRNA expression in a neuronal model of beta-amyloid toxicity. Cell Biol Toxicol 26: 367-377, 2010.

4. Sethi P, Jyoti A, Hussain E and Sharma D: Curcumin attenuates aluminium-induced functional neurotoxicity in rats. Pharmacol Biochem Behav 93: 31-39, 2009.

5. Han DW: Intestinal endotoxemia and liver disease-IETM theory of liver failure. Chin J Hepatol 3: 134-137, 1995.

6. Han DW: Intestinal endotoxemia as a pathogenetic mechanism in liver failure. World J Gaotroenteral 8: 961-965, 2002.

7. Zhao LF and Han DW: Clinical significance of endotoxemia in liver diseases. Shijie Huaren Xiaohua Zazhi 7: 391-393, 1999.

8. Zhou X, Han D, Xu R, Li S, Wu H, Qu C, Wang F, Wang X and Zhao Y: A model of metabolic syndrome and related diseases with intestinal endotoxemia in rats fed a high fat and high sucrose diet. PLoS One 9: e115148, 2014.

9. Yang W, Shi L, Chen L, Zhang B, Ma K, Liu Y and Qian Y: Protective effects of perindopril on d-galactose and aluminum trichloride induced neurotoxicity via the apoptosis of mitochondria-mediated intrinsic pathway in the hippocampus of mice. Brain Res Bull 109: 46-53, 2014.

10. Navarrete M, Núñez H, Ruiz S, Soto-Moyano R, Valladares L, White A and Pérez H: Prenatal undernutrition decreases the sensitivity of the hypothalamo-pituitary-adrenal axis in rat, as revealed by subcutaneous and intra-paraventricular dexamethasone challenges. Neurosci Lett 419: 99-103, 2007.

11. Coria F, Castaño EM and Frangione B: Brain amyloid in normal aging and cerebral amyloid angiopathy is antigenically related Alzheimer's disease beta-protein. Am J Pathol 129: 422-428, 1987.

12. Davies L, Wolska B, Hilbich C, Multhaup G, Martins R, Simms G, Beyreuther K and Masters CL: A4 amyloid protein deposition and the diagnosis of Alzheimer's disease: Prevalence in aged brains determined by immunocytochemistry compared with conventional neuropathologic techniques. Neurology 38 : $1688-1693,1988$
13. Kitamoto T, Ogomori K, Tateishi J and Prusiner SB: Formic acid pretreatment enhances immunostaining of cerebral and systemic amyloids. Lab Invest 57: 230-236, 1987.

14. Philippens IH, Ormel PR, Baarends G, Johansson M, Remarque EJ and Doverskog M: Acceleration of amyloidosis by inflammation in the amyloid-beta marmoset monkey model of Alzheimer's disease. J Alzheimers Dis 55: 101-113, 2017.

15. Perl DP and Pendlebury WW: Aluminum neurotoxicity-potential role in the pathogenesis of neurofibrillary tangle formation. Can J Neurol Sci 13 (4 Suppl): S441-S445, 1986.

16. Kawahara M, Muramoto K, Kobayashi K, Mori H and Kuroda Y: Aluminum promotes the aggregation of Alzheimer's amyloid beta-protein in vitro. Biochem Biophys Res Commun 198: 531-535, 1994.

17. Prakash D, Gopinath K and Sudhandiran G: Fisetin enhances behavioral performances and attenuates reactive gliosis and inflammation during aluminum chloride-induced neurotoxicity. Neuromolecular Med 15: 192-208, 2013.

18. Andreasen AS, Krabbe KS, Krogh-Madsen R, Taudorf S, Pedersen BK and Møller K: Human endotoxemia as a model of systemic inflammation. Curr Med Chem 15: 1697-1705, 2008.

19. Liu C, Cui Z, Wang S and Zhang D: CD93 and GIPC expression and localization during central nervous system inflammation. Neural Regen Res 9: 1995-2001, 2014.

20. Mandrekar-Colucci S and Landreth GE: Microglia and inflammation in Alzheimer's disease. CNS Neurol Disord Drug Targets 9: 156-167, 2010.

21. Skelly DT, Hennessy E, Dansereau MA and Cunningham C: A systematic analysis of the peripheral and CNS effects of systemic LPS, IL-1 $\beta$, [corrected] TNF- $\alpha$ and IL- 6 challenges in C57BL/6 mice. PLoS One 8: e69123, 2013.

22. Kang YH, Lee CH, Monroy RL, Dwivedi RS, Odeyale C and Newball HH: Uptake, distribution and fate of bacterial lipopolysaccharides in monocytes and macrophages: An ultrastructural and functional correlation. Electron Microsc Rev 5: 381-419, 1992.

23. Liu Y, Walter S, Stagi M, Cherny D, Letiembre M, Schulz-Schaeffer W, Heine H, Penke B, Neumann H and Fassbender K: LPS receptor (CD14): A receptor for phagocytosis of Alzheimer's amyloid peptide. Brain 128: 1778-1789, 2005.

24. Jaeger LB, Dohgu S, Sultana R, Lynch JL, Owen JB, Erickson MA, Shah GN, Price TO, Fleegal-Demotta MA, Butterfield DA and Banks WA: Lipopolysaccharide alters the blood-brain barrier transport of amyloid beta protein: A mechanism for inflammation in the progression of Alzheimer's disease. Brain Behav Immun 23: 507-517, 2009.

25. Wu L, Zhang K, Hu G, Yan H, Xie C and Wu X: Inflammatory response and neuronal necrosis in rats with cerebral ischemia. Neural Regen Res 9: 1753-1762, 2014.

26. Zaky A, Mohammad B, Moftah M, Kandeel KMand Bassiouny AR: Apurinic/apyrimidinic endonuclease 1 is a key modulator of aluminum-induced neuroinflammation. BMC Neurosci 14: 26, 2013.

27. Nedzvetsky VS, Tuzcu M, Yasar A, Tikhomirov AA and Baydas G: Effects of vitamin $\mathrm{E}$ against aluminum neurotoxicity in rats. Biochemistry (Mosc) 71: 239-244, 2006.

28. Tsunoda M and Sharma RP: Modulation of tumor necrosis factor alpha expression in mouse brain after exposure to aluminum in drinking water. Arch Toxicol 73: 419-426, 1999.

29. Mackenzie IR: Anti-inflammatory drugs and Alzheimer-type pathology in aging. Neurology 54: 732-734, 2000.

30. Samy AS and Igwe OJ: Regulation of IL-1 $\beta$-induced cyclooxygenase-2 expression by interactions of $\mathrm{A} \beta$ peptide, apolipoprotein $\mathrm{E}$ and nitric oxide in human neuroglioma. J Mol Neurosci 47: 533-545, 2012

31. Gutteridge JM, Quinlan GJ, Clark I and Halliwell B: Aluminium salts accelerate peroxidation of membrane lipids stimulated by iron salts. Biochim Biophys Acta 835: 441-447, 1985.

32. Wu CH, Wen CY, Shieh JY and Ling EA: Remodeling of membrane-bound glycoproteins containing alpha-D-galactose in the cerebral endothelial cells of rats during blood-brain barrier maturation and alteration. J Hirnforsch 38: 541-552, 1997. 